Marquette University

e-Publications@Marquette

Electrical and Computer Engineering Faculty

Electrical and Computer Engineering, Department

Research and Publications

7-1-2013

\title{
Robust and Resilient Finite-time Bounded Control of Discrete-time Uncertain Nonlinear Systems
}

Mohammad N. ElBsat

Marquette University

Edwin E. Yaz

Marquette University, edwin.yaz@marquette.edu

Accepted version. Automatica, Vol. 49, No. 7 (July 2013): 2292-2296. DOI. (C) 2013 Elsevier Ltd. Used with permission. 


\title{
e-Publications@Marquette
}

\section{Electrical and Computer Engineering Faculty Research and Publications/College of Engineering}

This paper is NOT THE PUBLISHED VERSION; but the author's final, peer-reviewed manuscript. The published version may be accessed by following the link in th citation below.

Automatica, Vol. 49, No. 7 (July 2013): 2292-2296. DOl. This article is (C Elsevier and permission has been granted for this version to appear in e-Publications@Marquette. Elsevier does not grant permission for this article to be further copied/distributed or hosted elsewhere without the express permission from Elsevier.

\section{Robust and Resilient Finite-Time Bounded Control of Discrete-Time Uncertain Nonlinear Systems}

\author{
Mohammad N. ElBsat
}

Electrical and Computer Engineering Department, Marquette University, Milwaukee, WI Edwin E. Yaz

Electrical and Computer Engineering Department, Marquette University, Milwaukee, WI

\begin{abstract}
Finite-time state-feedback stabilization is addressed for a class of discrete-time nonlinear systems with conictype nonlinearities, bounded feedback control gain perturbations, and additive disturbances. First, conditions for the existence of a robust and resilient linear state-feedback controller for this class of systems are derived. Then, using linear matrix inequality techniques, a solution for the controller gain and the maximum allowable bound on the gain perturbation is obtained. The developed controller is robust for all unknown nonlinearities lying in a known hypersphere with an uncertain center and all admissible disturbances. Moreover, it is resilient against any bounded perturbations that may alter the controller's gain by at most a prescribed amount. The paper is concluded with a numerical example showcasing the applicability of the main result.
\end{abstract}




\section{Keywords}

Discrete-time systems, LMIs, Nonlinear systems, State-feedback, Finite-time stability, Robust Control

\section{Introduction}

Finite-time stabilization via state-feedback of discrete-time nonlinear systems with additive disturbances and unknown nonlinearities lying within a hypersphere of uncertain center is presented. A system is said to be FiniteTime Stable (FTS) or more precisely Finite-Time Bounded (FTB) if, given a bound on the initial state of the system and the disturbance input, the state of the system does not exceed a given bound over a fixed time interval and for all admissible disturbances. Various developments and extensions in the field of FTS have been implemented, most of which have been applied to linear systems (Amato and Ariola, 2005, Amato et al., 2010a, Amato et al., 2010b, Amato et al., 2004, Dorato et al., 1997, Garcia et al., 2009, Zhang and An, 2008, only to mention a few due to space limitations). However, to the best of our knowledge, the study of FTS or FTB of nonlinear systems is rarely addressed in the literature. Some of the work related to FTB of nonlinear systems is found in Amato, Cosentino, and Merola (2010), Yang, Li, and Chen (2009), and Zhuang and Liu (2010). In ElBsat and Yaz (2011), a robust and resilient FTB controller design for a class of discrete-time nonlinear systems with conic-type nonlinearities lying in a hypersphere with a known center, feedback gain perturbations, and additive disturbances is presented.

The work presented in this technical communique is an extension of that in ElBsat and Yaz (2011). Here, the center of the hypersphere, which contains the set of unknown nonlinear vector functions, is described by a linear system with uncertainty in its dynamics. Moreover, an analysis of the upper bound on the gain perturbation vector as a function of the vector's direction in a three-dimensional (3D) space is presented. The significance of the controller design developed here is that it requires the knowledge of a dynamical bound on the system's nonlinearity rather than its exact dynamics. Thus, the controller design developed is applicable to all nonlinear systems which are locally Lipschitz (Khalil, 2002). Furthermore, the controller is robust for all nonlinearities lying within the conic bound, all admissible disturbances, and all bounded perturbations affecting the center of the conic bound. It is also resilient against all bounded perturbations which may affect its gain and which may occur as a result of computational or implementation errors (Takabashi, Dutra, Palhares, \& Peres, 2000).

Next, the system and controller models are introduced. In Section $\underline{3}$, the main results of this communique are presented followed by a simulation study illustrating the use of these results. Moreover, a study of the bound on the gain perturbation vector as a function of the vector's direction in a 3D space is introduced.

The following notation is used: $x \in R^{n}$ is an $n$-dimensional vector, $\|x\|=\left(x^{T} x\right)^{1 / 2}$ is the Euclidean norm, $(\cdot)^{T}$ and $(\cdot)^{-1}$ are the matrix transpose and inverse operators, respectively, $A \in R^{m \times n}$ is an $m \times n$ real matrix, $A>0(A<0)$ is a positive-definite (negative-definite) matrix, $I$ is an identity matrix of appropriate dimensions, and $\mathrm{N}_{0}$ is the set of nonnegative integers.

\section{Definition: finite-time boundedness}

Generally, a system is said to be Finite-Time Bounded (FTB) if, given a bound on the initial state of the system and the disturbance input, the state of the system does not exceed a given bound over a fixed time interval and for all admissible additive disturbances. In this work, the definitions stated in the work of Amato and Ariola (2005) are adopted and are generalized to include nonlinear systems. Consider a system that is described by the following dynamics:

(1) $x_{k+1}=f\left(x_{k}, u_{k}, w_{k}\right)$

where $f$ is the vector function which is in general nonlinear. 
System (1) is said to be FTB with respect to $\left(\alpha_{x}, \alpha_{w}, \beta, R, N\right)$ where $R>0, \alpha_{w} \geq 0,0 \leq \alpha_{x} \leq \beta$ and $N \in N_{0}$ if

$$
\left\{\begin{array}{l}
x_{0}^{T} R x_{0} \leq \alpha_{x}^{2} \\
w_{0}^{T} w_{0} \leq \alpha_{w}^{2}
\end{array} \Rightarrow x_{k}^{T} R x_{k} \leq \beta^{2} \forall k=1, \ldots, N\right.
$$

\section{System and control model}

Consider the following discrete-time nonlinear system:

(2) $\left\{\begin{array}{c}x_{k+1}=f\left(x_{k}, u_{k}, w_{k}\right) \\ w_{k+1}=\Phi w_{k}\end{array}\right.$

where $x_{k} \in W_{n} \subset R^{n}$ is the state vector, $u_{k} \in W_{m} \subset R^{m}$ is the input vector, $w_{k} \in W_{r} \subset R^{r}$ is the disturbance input, and $\Phi \in R^{r \times r}$, and $W_{m}$ are open and connected sets. The disturbance is one of the known waveforms (Johnson, 1980), but it does not have to be of finite-energy type. $f\left(x_{k}, u_{k}, w_{k}\right)$ is an unknown nonlinear vector function whose dynamics have the following conic sector description:

(3) $\left\|f\left(x_{k}, u_{k}, w_{k}\right)-\left(A x_{k}+B u_{k}+F w_{k}\right)\right\| \leq\left\|C_{f} x_{k}+D_{f} u_{k}+F_{f} w_{k}\right\|$

for all time $k \in N_{0}, x_{k} \in W_{n}, u_{k} \in W_{m}$, and $w_{k} \in W_{r}$ where $\tilde{A} \in R^{n \times n}, \tilde{B} \in R^{n \times m}$, and $\tilde{F} \in R^{n \times r}$, which are assumed to have the following expressions:

(4) $\left\{\begin{array}{l}\tilde{A}=A+A_{\Delta} \\ \tilde{B}=B+B_{\Delta} \text { such that } \\ \tilde{\sim} \\ F=F+F_{\Delta}\end{array}\left\{\begin{array}{l}A_{\Delta} A_{\Delta}^{T} \leq \sigma_{A}^{2} I \\ B_{\Delta} B_{\Delta}^{T} \leq \sigma_{B}^{2} I \\ F_{\Delta} F_{\Delta}^{T} \leq \sigma_{F}^{2} I .\end{array}\right.\right.$

The matrices $A_{\Delta}, B_{\Delta}$, and $F_{\Delta}$ are unknown bounded perturbations with known scalar upper bounds $\sigma_{A}, \sigma_{B}$, and $\sigma_{F}$, respectively. Matrices $A, B, F, C_{f}, D_{f}$, and $F_{f}$ are assumed to be known for the system in consideration. The inequality shown in (3) implies that the unknown nonlinearity lies in an $n$-dimensional hypersphere whose center is the linear system $A x_{k}+B u_{k}+F w_{k}$ with uncertain parameter matrices and whose radius is bounded by the right-hand side term of (3). Moreover, given system (2), a linear state-feedback controller with gain $K \in$ $R^{m \times n}$ is considered such that

(5) $u_{k}=K x_{k}$

which leads to the following closed-loop system:

(6) $\left\{\begin{array}{c}x_{k+1}=(\tilde{A}+\tilde{B} K) x_{k}+\tilde{F} w_{k}+\mathfrak{I}_{k} \\ w_{k+1}=\Phi w_{k}\end{array}\right.$

where $\mathfrak{I}_{k}=f\left(x_{k}, u_{k}, w_{k}\right)-\left(A x_{k}+B u_{k}+F w_{k}\right)$. 


\section{Main results}

The objective is to find a robust and resilient state-feedback controller that will render the closed-loop system FTB as long as the nonlinearity is within the hypersphere defined by (3). Theorem 1 states the conditions for the existence of a robust linear state-feedback controller for the class of nonlinear systems described by (2).

Theorem 1

Given sector condition (3), matrices $A, B, F, C_{f}, D_{f}$, and $F_{f}$, and the upper bounds $\sigma_{A}, \sigma_{B}$, and $\sigma_{F}$, system $(6)$ is FTB with respect to $\left(\alpha_{x}, \alpha_{w}, \beta, R, N\right)$, if there exist positive-definite matrices $Q_{1} \in R^{n \times n}$ and $Q_{2} \in R^{r \times r}$, a matrix $Y \in R^{m \times n}$, and positive scalars $\gamma \geq 1, b_{1}, \delta, \alpha_{1}, \alpha_{2}$, and $\alpha_{3}$ such that the following conditions hold:

(7) $M=M^{T}=\left[m_{i j}\right]_{i, i=1 \ldots 8}>0$

(8) $\left[\begin{array}{cc}Q_{1}-\delta R^{-1} & 0 \\ 0 & Q_{2}-\delta I\end{array}\right]>0$

(9) $\delta R^{-1} \frac{\beta^{2} \gamma^{-N}}{\alpha_{x}^{2}+\alpha_{w}^{2}}-Q_{1}>0$

Where

$$
\begin{aligned}
& m_{11}=\gamma Q_{1}, m_{13}=Q_{1} A^{T}+Y^{T} B^{T}, m_{14}=Q_{1} C_{f}^{T}+Y^{T} D_{f}^{T}, \\
& m_{16}=Q_{1}, m_{17}=Y^{T}, m_{22}=\gamma Q_{2}, m_{23}=Q_{2} F^{T}, m_{24}=Q_{2} F_{f}^{T}, \\
& m_{25}=Q_{2} \Phi^{T}, m_{28}=Q_{2}, \\
& m_{33}=Q_{1}-\left(b_{1}+\alpha_{1} \sigma_{A}^{2}+\alpha_{2} \sigma_{B}^{2}+\alpha_{3} \sigma_{F}^{2}\right) I, \\
& m_{44}=b_{1} I, m_{55}=Q_{2}, m_{66}=\alpha_{1} I, m_{77}=\alpha_{2} I, m_{88}=\alpha_{3} I,
\end{aligned}
$$

and the unspecified submatrices are equal to zero matrices with appropriate dimensions. The controller gain is given by $K=Y Q_{1}^{-1}$.

Sketch of Proof

Assume that $x_{0}^{T} R x_{0} \leq \alpha_{x}^{2}, w_{0}^{T} w_{0} \leq \alpha_{w}^{2}$, and that $x_{k}^{T} R x_{k} \leq \beta^{2} \forall k=1, \ldots, N$. Consider the energy function,

(10) $V_{k}=x_{k}^{T} P_{1} x_{k}+w_{k}^{T} P_{2} w_{k}$ such that $V_{k+1}<\gamma V_{k}$

where $P_{1}>0, P_{2}>0$, and $\gamma \geq 1$.

Moreover, consider the inequality shown in (3) which can be rewritten as follows:

(11) $\mathfrak{I}_{k}^{T} \mathfrak{I}_{k} \leq\left(A_{f} x_{k}+F_{f} w_{k}\right)^{T}\left(A_{f} x_{k}+F_{f} w_{k}\right)$

where $A_{f}=C_{f}+D_{f} K$.

From (10), replacing $x_{k+1}$ and $w_{k+1}$ with the equations of system (2), and applying Schur's complement the following matrix inequality is obtained.

(12) $\left[\begin{array}{ll}h_{11} & h_{12} \\ h_{12}^{T} & h_{22}\end{array}\right]>\left[\begin{array}{cc}0 & -\mathfrak{\Im}_{k}^{T} P_{1} \\ -P_{1} \Im_{k} & 0\end{array}\right]$ 
Where

$h_{11}=\gamma\left(x_{k}^{T} P_{1} x_{k}+w_{k}^{T} P_{2} w_{k}\right)-w_{k}^{T} \Phi^{T} P_{2} \Phi w_{k}, h_{22}=P_{1}$, and $h_{12}=\left(A_{c} x_{k}+F w_{k}\right)^{T} P_{1}$.

For any $b_{1}>0$, it is true that

$$
\left[\begin{array}{cc}
b_{1}^{-1} \mathfrak{J}_{k}^{T} \mathfrak{J}_{k} & 0 \\
0 & b_{1} P_{1}^{2}
\end{array}\right] \geq\left[\begin{array}{cc}
0 & -\mathfrak{J}_{k}^{T} P_{1} \\
-P_{1} \mathfrak{J}_{k} & 0
\end{array}\right]
$$

Using (13), the following is a sufficient condition for (12):

$\left[\begin{array}{ll}h_{11} & h_{12} \\ h_{12}^{T} & h_{22}\end{array}\right]>\left[\begin{array}{cc}b_{1}^{-1} \mathfrak{\Im}_{k}^{T} \mathfrak{\Im}_{k} & 0 \\ 0 & b_{1} P_{1}^{2}\end{array}\right]$

Moreover, based on (11), (14) will still be satisfied, if the following inequality holds.

$$
\left[\begin{array}{cc}
h_{11}-b_{1}^{-1}\left(A_{f} x_{k}+F_{f} w_{k}\right)^{T}\left(A_{f} x_{k}+F_{f} w_{k}\right) & h_{12} \\
h_{12}^{T} & h_{22}-b_{1} P_{1}^{2}
\end{array}\right]>0
$$

Applying Schur's complement to $\underline{(15)}$, substituting the expressions of $h_{11}, h_{12}$, and $h_{22}$, then rearranging the obtained expression in a quadratic format in $\left[\begin{array}{cc}x_{k}^{T} & w_{k}^{T}\end{array}\right]$ yields a positive-definite matrix, which can be rewritten as follows:

$$
\left[\begin{array}{cc}
\gamma P_{1}-b_{1}^{-1} A_{f}^{T} A_{f} & -b_{1}^{-1} A_{f}^{T} F_{f} \\
-b_{1}^{-1} F_{f}^{T} A_{f} & \gamma P_{2}-\Phi^{T} P_{2} \Phi-b_{1}^{-1} F_{f}^{T} F_{f}
\end{array}\right]-\left[\begin{array}{c}
A_{c}^{T} P_{1} \\
\tilde{F^{T} P_{1}}
\end{array}\right]\left(P_{1}-b_{1} P_{1}^{2}\right)^{-1}\left[\begin{array}{cc}
P_{1} A_{c} & P_{1} F
\end{array}\right]>0
$$

It is worth noting that the condition $P_{1}-b_{1} P_{1}^{2}>0$ due to Schur's complement will be implicitly satisfied and thus it would be redundant to consider it as one of the conditions for the existence of the robust controller developed.

By applying Schur's complement to $\underline{(16)}$, and after some algebraic manipulations and substituting for $A_{f}$ and $A_{c}$, we obtain

(17) $\left[\begin{array}{ccccc}\gamma Q_{1} & 0 & Q_{1} A^{T}+Y^{T} \bar{B}^{T} & Q_{1} C_{f}^{T}+Y^{T} D_{f}^{T} & 0 \\ * & \gamma Q_{2} & Q_{2} F^{T} & Q_{2} F_{f}^{T} & Q_{2} \Phi^{T} \\ * & * & Q_{1}-b_{1} I & 0 & 0 \\ * & * & * & b_{1} I & 0 \\ * & * & * & * & Q_{2}\end{array}\right]>0$

where $*$ denotes the elements that need to be added in order to have a symmetric matrix, $Q_{1}=P_{1}^{-1}, Q_{2}=P_{2}^{-1}$, and $Y=K Q_{1}$. 
Substituting (4) in (17), rearranging the obtained inequality and after showing that for any positive scalars $\alpha_{1}, \alpha_{2}$, and $\alpha_{3}$ :

$$
\left[\begin{array}{ccccc}
-\alpha_{1}^{-1} Q_{1}^{2}-\alpha_{2}^{-1} Y^{T} Y & 0 & -Q_{1} A_{\Delta}^{T}-Y^{T} B_{\Delta}^{T} & 0 & 0 \\
* & \alpha_{3}^{-1} Q_{2}^{2} & Q_{2} F_{\Delta}^{T} & 0 & 0 \\
* & * & \Psi & 0 & 0 \\
* & * & * & 0 & 0 \\
* & * & * & * & 0
\end{array}\right] \leq 0
$$

where $\Psi=\alpha_{1} A_{\Delta} A_{\Delta}^{T}+\alpha_{2} B_{\Delta} B_{\Delta}^{T}+\alpha_{3} F_{\Delta} F_{\Delta}^{T}$, we arrive at condition (7).

Now, we proceed to show the derivation of conditions (8), (9). Applying successive substitutions of (10)for $k=$ $1,2, \ldots, N$, knowing that $\gamma \geq 1$, replacing $V_{k}$ and $V_{0}$ with their corresponding expressions, and since $x_{k}^{T} P_{1} x_{k}<$ $x_{k}^{T} P_{1} x_{k}+w_{k}^{T} P_{2} w_{k}$, we obtain the following:

(19) $x_{k}^{T} P_{1} x_{k}<\gamma^{N}\left(x_{0}^{T} P_{1} x_{0}+w_{0}^{T} P_{2} w_{0}\right)$.

In (19), introduce the term $R^{1 / 2} R^{-1 / 2}$ to the left- and right-hand side of $P_{1}$, express the right-hand side of the inequality in a quadratic format, and apply Rayleigh's inequality, which states that given $Q>0$, then $\lambda_{\min }(Q) x_{k}^{T} x_{k}<x_{k}^{T} Q x_{k}<\lambda_{\max }(Q) x_{k}^{T} x_{k}$ is true, to obtain the following:

(20) $\lambda_{\min }\left(R^{-1 / 2} P_{1} R^{-1 / 2}\right) x_{k}^{T} R x_{k}<\gamma^{N} \lambda_{\max }\left(\left[\begin{array}{cc}R^{-1 / 2} P_{1} R^{-1 / 2} & 0 \\ 0 & P_{2}\end{array}\right]\right)\left(\alpha_{x}^{2}+\alpha_{w}^{2}\right)$.

In order for $x_{k}^{T} R x_{k}<\beta^{2}$ to be satisfied, then

(21) $\lambda_{\max }\left(\left[\begin{array}{cc}R^{-1 / 2} P_{1} R^{-1 / 2} & 0 \\ 0 & P_{2}\end{array}\right]\right)<\frac{\beta^{2} \gamma^{-N}}{\left(\alpha_{x}^{2}+\alpha_{w}^{2}\right)} \lambda_{\min }\left(R^{-1 / 2} P_{1} R^{-1 / 2}\right)$

must hold. Let $\delta^{-1}>0$ such that

(22) $\lambda_{\max }\left(\left[\begin{array}{cc}R^{-1 / 2} P_{1} R^{-1 / 2} & 0 \\ 0 & P_{2}\end{array}\right]\right)<\delta^{-1}$

and

(23) $\delta^{-1}<\frac{\beta^{2} \gamma^{-N}}{\left(\alpha_{x}^{2}+\alpha_{w}^{2}\right)} \lambda_{\min }\left(R^{-1 / 2} P_{1} R^{-1 / 2}\right)$.

Then, conditions (8), (9) can be obtained from (22), (23) respectively through some algebraic manipulations and the proof of Theorem 1 is concluded.

Next, the controller gain is assumed to have additive bounded perturbations and conditions for the existence of a finite-time controller that is not only robust but also resilient are derived. Consider system (6) with a controller gain $K$ instead of $K$ : 
(24) $\left\{\begin{array}{c}x_{k+1}=(\tilde{\sim} \tilde{A}+B) x_{k}+\tilde{F} w_{k}+\widetilde{I}_{k} \\ w_{k+1}=\Phi w_{k}\end{array}\right.$

where $\mathfrak{I}_{k}$ satisfies inequality $\underline{(11)}$ and

(25) $K=K_{r}+K_{\Delta}$

where $K_{r}$ is the controller gain and $K_{\Delta}$ is an unknown additive bounded gain perturbation with a scalar upper bound $\sigma_{K}$ such that

(26) $K_{\Delta}^{T} K_{\Delta} \leq \sigma_{K}^{2} I$

\section{Theorem 2}

Given sector condition (3), matrices $A, B, F, C_{f}, D_{f}$, and $F_{f}$, and the upper bounds $\sigma_{A}, \sigma_{B}$, and $\sigma_{F}$, system (24) is FTB with respect to $\left(\alpha_{x}, \alpha_{w}, \beta, R, N\right)$, if there exist positive-definite matrices $Q_{1} \in R^{n \times n}$ and $Q_{2} \in R^{r \times r}$, a matrix $Y_{r} \in R^{m \times n}$, and positive scalars $\gamma \geq 1, b_{1}, b_{2}, b_{3}, \alpha_{1}, \alpha_{2}, \alpha_{3}$ and $\delta$ such that conditions (8), (9), and the following hold:

(27) $Z=Z^{T}=\left[z_{i j}\right]_{i, j=1 \ldots 9}>0$

where

$$
\begin{aligned}
& z_{11}=\gamma Q_{1}, z_{13}=Q_{1} A^{T}+Y_{r}^{T} B^{T}, z_{14}=Q_{1} C_{f}^{T}+Y^{T} D_{f}^{T}, \\
& z_{16}=Q_{1}, z_{17}=Y_{r}^{T}, z_{19}=Q_{1}, z_{22}=\gamma Q_{2}, z_{23}=Q_{2} F^{T}, \\
& z_{24}=Q_{2} F_{f}^{T}, z_{25}=Q_{2} \Phi^{T}, z_{28}=Q_{2}, \\
& z_{33}=Q_{1}-\left(b_{1}+\alpha_{1} \sigma_{A}^{2}+\alpha_{2} \sigma_{B}^{2}+\alpha_{3} \sigma_{F}^{2}\right) I-b_{2} B B^{T}, \\
& z_{34}=-b_{2} B D_{f}^{T}, z_{37}=-b_{2} B, z_{44}=b_{1} I-b_{2} D_{f} D_{f}^{T}, \\
& z_{47}=-b_{2} D_{f}, Z_{55}=Q_{2}, z_{66}=\alpha_{1} I, z_{77}=\left(\alpha_{2}-b_{2}\right) I, \\
& z_{88}=\alpha_{3} I, z_{99}=b_{3} I
\end{aligned}
$$

and the unspecified submatrices are equal to zero matrices of appropriate dimensions. The controller gain is given by:

$$
\text { (28) } K_{r}=Y_{r} Q_{1}^{-1}
$$

The bound on the maximum allowable controller gain perturbation is given by:

$$
\text { (29) } \sigma_{K}=\sqrt{b_{2} b_{3}^{-1}}
$$

Consider Theorem 1 and replace $Y$ by $Y$ where $Y=K Q_{1}=Y_{r}+Y_{\Delta}, Y_{r}=K_{r} Q_{1}$, and $Y_{\Delta}=K_{\Delta} Q_{1}$. Then, (7) can be rewritten as the equivalent condition 
(30) $L>\left[\begin{array}{cccccccc}0 & 0 & -Y_{\Delta}^{T} B^{T} & -Y_{\Delta}^{T} D_{f}^{T} & 0 & 0 & -Y_{\Delta}^{T} & 0 \\ * & 0 & 0 & 0 & 0 & 0 & 0 & 0 \\ * & * & 0 & 0 & 0 & 0 & 0 & 0 \\ * & * & * & 0 & 0 & 0 & 0 & 0 \\ * & * & * & * & 0 & 0 & 0 & 0 \\ * & * & * & * & * & 0 & 0 & 0 \\ * & * & * & * & * & * & 0 & 0 \\ * & * & * & * & * & * & * & 0\end{array}\right]$

where $L$ is the same matrix as $M$ with $Y$ evaluated at $Y_{r}, L=\left.M\right|_{Y=Y_{r}}$.

For an arbitrary $b_{2}>0$, it is true that

$$
\left[\begin{array}{c}
b_{2}^{-1 / 2} Y_{\Delta}^{T} \\
0 \\
b_{2}^{1 / 2} B \\
b_{2}^{1 / 2} D_{f} \\
0 \\
0 \\
b_{2}^{1 / 2} I \\
0
\end{array}\right]\left[\begin{array}{c}
b_{2}^{-1 / 2} Y_{\Delta}^{T} \\
0 \\
b_{2}^{1 / 2} B \\
0 \\
0 \\
b_{2}^{1 / 2} I \\
0
\end{array}\right] \geq 0
$$

Using (31), the following condition is sufficient for $(30)$ to hold.

$(32) L>\left[\begin{array}{cccccccc}b_{2}^{-1} Y_{\Delta}^{T} Y_{\Delta} & 0 & 0 & 0 & 0 & 0 & 0 & 0 \\ * & 0 & 0 & 0 & 0 & 0 & 0 & 0 \\ * & * & b_{2} B B^{T} & b_{2} B D_{f}^{T} & 0 & 0 & b_{2} B & 0 \\ * & * & * & b_{2} D_{f} D_{f}^{T} & 0 & 0 & b_{2} D_{f} & 0 \\ * & * & * & * & 0 & 0 & 0 & 0 \\ * & * & * & * & * & 0 & 0 & 0 \\ * & * & * & * & * & * & b_{2} I & 0 \\ * & * & * & * & * & * & * & 0\end{array}\right]$.

Now, substituting the expression of $Y_{\Delta}$ in (32), using (26), applying some algebraic manipulations, and letting $b_{3}=b_{2} \sigma_{K}^{-2}$, it can be shown that if (27) holds, (32) still holds. The derivation of conditions (8), (9)is the same as that shown in the proof of Theorem 1 . This concludes the proof of Theorem 2.

Given $\left(\alpha_{x}, \alpha_{w}, \beta, R, N\right)$, system $(24)$, matrices $A, B, F, C_{f}, D_{f}$, and $F_{f}$, the upper bounds $\sigma_{A}, \sigma_{B}$, and $\sigma_{F}$, and for a fixed value of $\gamma$, conditions (27), (8), (9) constitute a set of LMIs with unknown variables $Q_{1}, Q_{2}, b_{1}, b_{2}, b_{3}, \alpha_{1}, \alpha_{2}, \alpha_{3}$, and $Y_{r}$. Thus, a controller gain and a bound on the gain perturbation for which the LMIs are feasible can be solved for and obtained from (28), (29) respectively. In the next section, a numerical example is provided to illustrate the applicability of the developed controller design. 


\section{Simulation studies}

\subsection{Application to Chua's circuit}

Consider the following closed-loop system based on the discretized state-space model corresponding to Chua's circuit (Chua, Wu, Hung, \& Zhong, 1993).

(33) $\left\{\begin{array}{c}x_{k+1}=\tilde{A} x_{k}+\tilde{B} u_{k}+\tilde{F} w_{k}+\widetilde{I}_{k} \\ w_{k+1}=\Phi w_{k}\end{array}\right.$

where $A, B$, and $F$ are given by (4),

$A=\left[\begin{array}{ccc}1-T \alpha_{c}(1+b) & T \alpha_{C} & 0 \\ T & 1-T & T \\ 0 & -T \beta_{c} & 1-T \mu\end{array}\right], B=T\left[\begin{array}{lll}2 & 5 & 4\end{array}\right]^{T}, x_{k}=\left[\begin{array}{lll}x_{k}^{1} & x_{k}^{2} & x_{k}^{3}\end{array}\right]^{T}, F=$

$T\left[\begin{array}{lll}1 & 1 & 1\end{array}\right]^{T}, \Phi=0.99$,

and

$\mathfrak{I}_{k}=0.5 T \alpha_{c}(a-b)\left(\left|x_{k}^{1}+1\right|-\left|x_{k}^{1}-1\right|\right)\left[\begin{array}{lll}1 & 0 & 0\end{array}\right]^{T}$

$x_{k}^{i}$ is the $i$ th state variable at time instant

$k, \alpha_{c}=9.1, \beta_{c}=16.5811, \mu=0.138083, a=-1.3659, b=-0.7408$ (Azemi \& Yaz, 2001), and $T=0.05 \mathrm{~s}$ is the sampling period. Since $\left|x_{k}^{1}+1\right|-\left|x_{k}^{1}-1\right| \leq\left|2 x_{k}^{1}\right|$, then $\mathfrak{\Im}_{k}^{T} \mathfrak{J}_{k} \leq\left(T \alpha_{c}(a-b) x_{k}^{1}\right)^{2}$, from which the values of $C_{f}, D_{f}$, and $F_{f}$ in $(3)$ can be easily derived. Moreover, the upper bound values on $A_{\Delta}, B_{\Delta}$, and $F_{\Delta}$ are considered to be $\sigma_{A}=0.02, \sigma_{B}=0.02$, and $\sigma_{F}=0.03$, respectively. Therefore, consider the following:

$A_{\Delta}=0.008 I, B_{\Delta}=\left[\begin{array}{lll}0 & 0.019 & 0\end{array}\right]^{T}, F_{\Delta}=\left[\begin{array}{lll}0 & 0.029 & 0\end{array}\right]^{T}$.

Given $\left(\alpha_{x}, \alpha_{w}, \beta, R, N\right)$, the feasibility of the LMIs while varying $\gamma^{-1}$ over the range $(0,1]$ is examined. A solution exists as long as there exists at least one value of $\gamma$ for which the LMIs are feasible. Otherwise, the design parameters must be modified. In this work, the simulations are conducted in MATLAB ${ }^{\circ}$ (R2010a), and the LMIs obtained are solved using the Robust Control Toolbox ${ }^{\mathrm{TM}}$ V3.4.1 in the MATLAB ${ }^{\circ}$ version indicated. It is worth noting that as the order of the system increases, it is inherent that the dimensions of the relevant LMIs will increase. However, with the ongoing advances in computational capabilities and speed of computers, an increase in the dimensions of the LMls to be solved does not present any computational extensiveness of the approach.

For the system considered and the set of parameters $\alpha_{x}=1.5, \alpha_{w}=1, \beta=11, R=I, N=20$, a solution for the controller gain is found for $\gamma=1.002$ where

$K_{r}=\left[\begin{array}{lll}-3.3782 & -3.4785 & -0.1466\end{array}\right]$ and $\sigma_{K}=0.021$.

System (33) is simulated with the perturbed controller applied during the first $N=20$ time steps then removed. Fig. 1 shows the norm of the state of the system with respect to time in both the closed-loop and open-loop cases. The norm of the state remains within the prescribed bound $\beta=11$ for every time step over the interval during which the controller is applied, despite the perturbations in its gain. Thus, the controller 
developed is performing as expected during the finite-time interval, and the system returns to its controller-free dynamics afterwards.

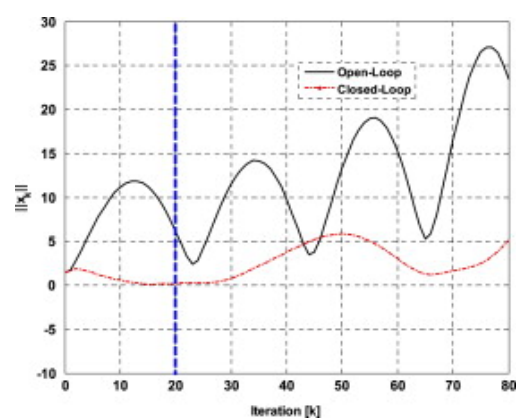

Fig. 1. Evolution of $\|x k\|$ over time for the open-loop and closed-loop cases. The vertical dashed line indicates where the controller is removed.

\subsection{Controller gain perturbation magnitude analysis}

In this section, the maximum allowable perturbation in the gain of the controller developed as a function of the position of the perturbation vector in a 3D space is examined. Condition $\underline{(26)}$ on the controller gain perturbation vector implies that $\left\|K_{\Delta}\right\|$ must be less than or equal to $\sigma_{K}$ in the case of a 3D system with a single input.

Therefore, in a 3D space, the solution for $\sigma_{K}$ obtained earlier would be the minimum norm of the perturbation vector for every direction of $K_{\Delta}$. However, this also means that, in certain directions, $\left\|K_{\Delta}\right\|$ may have a maximum value, which implies a possibility of a higher upper bound on the allowable perturbations in the controller gain.

In order to determine $\left\|K_{\Delta}\right\|$ as a function of its direction, $K_{\Delta}$ is expressed in spherical coordinates as shown below.

(34) $K_{\Delta}=K_{\Delta}[\sin \theta \cos \phi \quad \sin \theta \sin \phi \quad \cos \theta]$

where $K_{\Delta}=\left\|K_{\Delta}\right\|,-90^{\circ} \leq \theta \leq+90^{\circ}$, and $-180^{\circ} \leq \phi \leq+180^{\circ}$.

Moreover, conditions (7), (8), (9) are feasible for the solution of the unknown variables, $Q_{1}, Q_{2}, Y=$ $Y_{r}, b_{1}, \delta, \alpha_{1}, \alpha_{2}$, and $\alpha_{3}$ obtained from (27), (8), (9) with the value of $K_{r}$ assigned to $K$ and, consequently, the value of $Y_{r}$ assigned to $Y$. With all the variables in (7) known, the controller gain is perturbed by adding $K_{\Delta}$ to it.

For a fixed direction of $K_{\Delta}$ (i.e. one set of values of $\theta$ and $\left.\phi\right), K_{\Delta}$ is incrementally increased until (7) is no longer feasible. Then, the values of $\theta$ and $\phi$ are varied, and the previous steps are repeated until the ranges of $\theta$ and $\phi$ are covered.

The result obtained is shown in Fig. 2. The minimum value of $K_{\Delta}$ is 0.022 , which corresponds to a $4.55 \%$ difference from the value obtained for $\sigma_{K}$ using the inequalities developed in Theorem 2.

Furthermore, Fig. 2 shows that, for example in the direction of $\phi=75^{\circ}$ and $\theta=87^{\circ}$, the controller gain can be perturbed up to 1.305. Even though this result may reflect conservativeness in the results given in Theorem 2, it still shows that the controller design obtained is resilient against perturbations, whose upper bound is at least given by $\sigma_{K}$. 


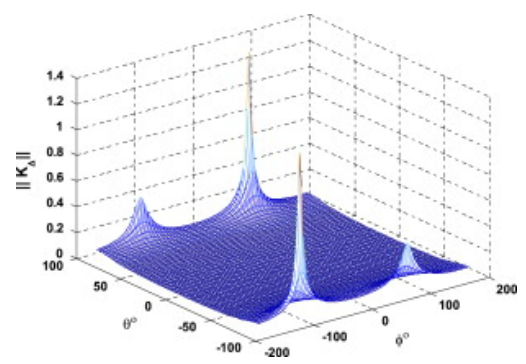

Fig. 2. Norm of the controller gain perturbation vector $K_{\Delta}$ as a function of its position in 3D space.

\section{Conclusion}

A robust and resilient FTB controller design is developed for a class of nonlinear systems with conic-type nonlinearities of uncertain center, known waveform type disturbances, and additive gain perturbations. A solution for the controller gain and the bound on the maximum allowable gain perturbation is obtained using LMI techniques. It is worth noting that the conditions arrived at in this paper reduce to the conditions existing in the literature on the finite-time bounded control of discrete-time linear systems. This fact can be shown by setting the right-hand side term of the conic sector condition and the bounds on the perturbations to zero. Thus, the class of nonlinear systems considered here and the associated results serve as a generalization of previous results. This is due to the fact that this class of systems, in addition to representing several nonlinearities that arise in the control literature, represents simple discrete-time linear systems considered by others in previous works.

\section{References}

Amato and Ariola, 2005 F. Amato, M. Ariola Finite-time control of discrete-time linear systems IEEE Transactions of Automatic Control, 50 (5) (2005), pp. 724-729

Amato et al., 2010a F. Amato, M. Ariola, C. Cosentino Finite-time stability of linear time-varying systems: analysis and controller design IEEE Transactions on Automatic Control, 55 (4) (2010), pp. 1003-1008

Amato et al., 2010b F. Amato, M. Ariola, C. Cosentino Finite-time control of discrete-time linear systems: analysis and design Automatica, 46 (5) (2010), pp. 919-924

Amato et al., 2004 Amato, F., Carbone, M., Ariola, M., \& Cosentino, C. (2004). Finite-time stability of discretetime systems. In Proceedings of American control conference, vol. 2 (pp. 1440-1444). Boston, Massachusetts.

Amato, Cosentino et al., 2010 F. Amato, C. Cosentino, A. Merola Sufficient conditions for finite-time stability and stabilization of nonlinear quadratic systems IEEE Transactions on Automatic Control, 55 (2) (2010), pp. 430-434

Azemi and Yaz, 2001 Azemi, A., \& Yaz, E.E. (2001). Full and reduced-order-robust adaptive observers for chaotic synchronization. In Proceedings of American control conference, vol. 3(pp. 1985-1990). Arlington, Virginia.

Chua et al., 1993 L. Chua, C. Wu, A. Hung, G. Zhong A universal circuit for studying and generating chaos-part I: routes to chaos IEEE Transactions on Circuits and Systems I: Fundamental Theory and Applications, 40 (10) (1993), pp. 732-744

Dorato et al., 1997 Dorato, P., Abdallah, C.T., \& Famularo, D. (1997). Robust finite-time stability design via linear matrix inequalities. In Proceedings of the 36th conference on decision \& control (pp. 1305-1306). San Diego, California.

ElBsat and Yaz, 2011 ElBsat, M.N., \& Yaz, E.E. (2011). Robust and resilient finite-time control of discrete-time nonlinear systems. In 18th IFAC world congress(pp. 6454-6459). Milan, Italy. Preprint.

Garcia et al., 2009 G. Garcia, S. Tarbouriech, J. Bernussou Finite-time stabilization of linear time-varying continuous systems IEEE Transactions on Automatic Control, 54 (2) (2009), pp. 364-369

Johnson, 1980 C.D. Johnson Disturbance-accommodation control: an overview of the subject Journal of Interdisciplinary Modeling and Simulation, 3 (1) (1980), pp. 1-29

Khalil, 2002 H.K. Khalil Nonlinear systems (2nd ed.), Prentice Hall, New Jersey (2002) 
Takabashi et al., 2000 Takabashi, R.H.C., Dutra, D.A., Palhares, R.M., \& Peres, P.K.D. (2000). On robust nonfragile static state-feedback controller synthesis. In Proceedings of the conference on decision and control (pp. 4909-4914). Sydney, Australia.

Yang et al., 2009 Y. Yang, J. Li, G. Chen Finite-time stability and stabilization of nonlinear stochastic hybrid systems Journal of Mathematical Analysis and Applications, 356 (1) (2009), pp. 338-345

Zhang and An, $2008 \mathrm{~W}$. Zhang, X. An Finite-time control of linear stochastic systems International Journal of Innovative Computing, Information and Control, 4 (3) (2008), pp. 687-694

Zhuang and Liu, 2010 Zhuang, J., \& Liu, F. (2010). Finite-time stabilization of a class of uncertain nonlinear systems with time-delay, In Proceedings of 7th conference on fuzzy systems and knowledge discovery (pp. 163-167). Yantai, China.

犃 The material in this paper was partially presented at the 18th IFAC World Congress, August 28-September 2, 2011, Milano, Italy. This paper was recommended for publication in revised form by Associate Editor Zongli Lin under the direction of Editor André L. Tits. 